\title{
Representation of brief temporal patterns, Hebbian synapses, and the left-hemisphere dominance for phoneme recognition
}

\author{
ROBERT MILLER \\ University of Otago, Dunedin, New Zealand
}

\begin{abstract}
There is considerable evidence, including that from single-unit classical conditioning studies, that patterns that are briefly extended in time can be learned. Learning of simultaneous patterns is usually assumed to occur by strengthening of selected synaptic connections converging on single neurons. The present paper extends this principle to the learning of patterns that are slightly extended in time. Evidence is reviewed that cortico-cortical axonal connections impose a range of conduction delays sufficient to permit temporal convergence at the single-neuron level between signals originating up to 100-200 msec apart. By selection of appropriate connections, brief temporal patterns can be represented by converging connections with different conduction delays. The Hebbian postulate for synaptic modification is sufficiently precise in time to permit such selection. Finally, this hypothesis is applied to the learning of phoneme recognition in the human forebrain, and a suggestion is made concerning the biological basis of left-hemisphere dominance for phoneme recognition.
\end{abstract}

In the last 30 years there has been great progress in the analysis of the neural mechanisms underlying pattern recognition. This field has been dominated by research on visual systems, particularly the visual cortex of the mammal, and has led to detailed documentation of visual pattern recognition in the adult and the processes of developmental plasticity by which the visual areas of the cortex acquire the capacity for pattern recognition.

The visual system is very highly developed for the analysis of patterns that are distributed through space. However, it is undoubtedly true that we have an ability to recognize patterns that are somewhat dispersed in time, although this is shown less by the visual system than by the auditory system. For instance, we can readily distinguish between different consonant phonemes, or the sounds of different musical instruments. Consonant phonemes are speech sounds consisting of brief sequences of tones whose components have different frequency characteristics (Tallal \& Schwartz, 1980). The distinguishing of one musical instrument from another depends largely on the "attack," that is, the characteristic sequence of frequencies at the beginning of each note (Taylor, 1969). In either case, we are distinguishing on the basis of different sequences of acoustic frequencies, compressed within an interval of the order of 100 msec.

The emphasis on visual cortical plasticity has been accompanied by a relative neglect of the study of the process of pattern recognition for patterns that are dispersed in

I thank Jeff Wickens and Robin Harvey for helpful comments during the preparation of this manuscript. This work was supported by the Neurological Foundation of New Zealand. Address requests for reprints to Robert Miller, Department of Anatomy and Neuroscience Centre, University of Otago, P.O. Box 913, Dunedin, New Zealand. time, and of the plastic processes by which temporal pattern recognition ability is acquired. This relative neglect applies not only to empirical investigations, but also to development of theoretical concepts. As an instance of the latter, modern interest has focused on the Hebbian paradigm for synaptic modification (Hebb, 1949) as a mechanism for associating simultaneous signals. Relatively little attention has been paid to the question of whether the Hebbian paradigm for association of correlated signals may also apply to signals originating at slightly different times.

Recognition of simultaneous visual patterns depends on spatial convergence/divergence in the connections relaying sensory signals to the visual cortex. However, in the older literature, concepts of temporal convergence/divergence are as well established as those of spatial convergence/divergence. It is therefore worth considering that the comparable process of recognition of patterns that are somewhat dispersed in time depends on temporal convergence/divergence in neural pathways.

My first aim in this paper is to review evidence showing that neural signals reaching the mammalian cerebral cortex are spread throughout the cortex along connections having substantially different conduction velocities (see also Miller, 1981). Therefore, a single cortical neuron can receive simultaneous converging inputs that originate at the periphery significantly dispersed in time. Thus, potentially, the network of direct (monosynaptic) corticocortical connections can encode brief temporal patterns (spread over an interval of the order of $100 \mathrm{msec}$ ). Second, I intend to show that Hebbian processes can serve to select appropriate connections from among such an array of temporally dispersed synaptic influences. Single neurons should therefore be able to acquire the ability to 
recognize such brief temporal patterns. Finally, I will advance a hypothesis that uses this mechanism to account for a particular specialization of the learning capability in humans, namely, the left hemisphere's superiority for recognition of consonant phonemes (speech sounds).

\section{Caliber of Cortico-Cortical Axonal Connections}

Ultrastructural studies of cerebral white matter (e.g., that of the corpus callosum) in various species have shown that a significant proportion of the axons therein are either very fine myelinated fibers or unmyelinated ones (Fleischhauer \& Wartenberg, 1967; Haug, Kölln, \& Rast, 1976; Naito, Miyakawa, \& Ito, 1971; Remahl \& Hildebrand, 1982; Seggie \& Berry, 1972; Swadlow, Waxman, \& Geschwind, 1980; Waxman \& Swadlow, 1976; Wiggins, Bissell, Durham, \& Samorajski, 1983). Fleischhauer and Wartenberg (1967) found that $40 \%$ of axons in the corpus callosum of the adult cat were unmyelinated; the median diameter of this population of axons was about $0.25 \mu \mathrm{m}$. Remahl and Hildebrand (1982) found that $15 \%-20 \%$ of axons in the adult cat corpus callosum were unmyelinated, with calibers ranging from 0.1 to $0.5 \mu \mathrm{m}$ (median about $0.25 \mu \mathrm{m}$ ). The majority of myelinated axons were under $1 \mu \mathrm{m}$ in diameter, with some having diameters as small as $0.3 \mu \mathrm{m}$. Haug et al. (1976) found that myelinated axons within or beneath the visual cortex of the adult cat had a median diameter of $0.8 \mu \mathrm{m}$, with more than $75 \%$ of the population less than $1 \mu \mathrm{m}$ in diameter, and the smallest $0.3 \mu \mathrm{m}$ in diameter. Naito et al. (1971) failed to find unmyelinated axons in the adult cat corpus callosum; the most common diameter for the myelinated fibers there was given as 0.8-1.0 $\mu \mathrm{m}$. Waxman and Swadlow (1976) found in the visual part of the rabbit corpus callosum that $45 \%$ of axons were unmyelinated, with calibers of 0.05-0.64 $\mu \mathrm{m}$ (median about $0.2 \mu \mathrm{m}$ ). The vast majority of myelinated axons in that study were under $1 \mu \mathrm{m}$ in diameter. Wiggins et al. (1983) found that approximately $20 \%$ of axons in the 35-day-old normal rat were unmyelinated. They reported that over $70 \%$ of the myelinated axons had circumferences of less than $3 \mu \mathrm{m}$ (roughly equivalent to a diameter of $1 \mu \mathrm{m}$ ).

\section{Conduction Velocity and Conduction Delays in Fine-Caliber Central Axons}

The conduction velocity of such fine-caliber axons may be expected to be very low. Available data from singleunit studies using antidromic invasion methods suggest that the slowest-conducting central axons have conduction velocities as low as $0.2-0.3 \mathrm{~m} / \mathrm{sec}$ (Ferreya Moyano \& Molina, 1980; Lee, Chung, Chung, \& Coggeshall, 1986; Swadlow \& Waxman, 1976; Willey, Maeda, \& Rafuse, 1975). Among a population of neurons projecting into the corpus callosum, Swadlow and Weyand (1981) found that 18 of 50 antidromic unit responses had axonal conduction velocities below $1 \mathrm{~m} / \mathrm{sec}$. Swadlow and Waxman (1976) argued that conduction velocities below $0.8 \mathrm{~m} / \mathrm{sec}$ are typical of the population of central unmyelinated axons. Lee et al. (1986) provided direct empirical correlation of conduction velocity and axonal caliber for axons in the dorsal columns of the cat spinal cord. Axons of $1 \mu \mathrm{m}$ caliber had a mean conduction velocity of about $0.7 \mathrm{~m} / \mathrm{sec}$, whereas those of $0.5 \mu \mathrm{m}$ caliber had a conduction velocity of about $0.5 \mathrm{~m} / \mathrm{sec}$.

Whether myelinated or unmyelinated, axons of this caliber may be expected to impose quite long conduction delays between soma and synapse. This supposition is also confirmed by electrophysiological studies in which single units are antidromically invaded from other cortical sites. A small proportion of antidromic responses in cortical units projecting across the corpus callosum or to cortical areas on the same side have been reported to have latencies longer than $40 \mathrm{msec}$ in the rabbit (Swadlow, 1974) and in the cat (Miller, 1976).

It is probable that, in the populations of neurons studied by the above authors, the contribution of axons having long conduction delays from soma to synapse was underestimated: Microelectrodes tend to be selective for larger neurons with larger axons. Although this is true to a lesser extent for tungsten microelectrodes, such as those used in the studies of Swadlow (1974), Swadlow and Waxman (1976), and Miller (1976), than for glass microelectrodes, even tungsten microelectrodes probably neglect to some extent the smaller neurons with very fine axons. Evidence for this underrepresentation can be found if the conduction-velocity criteria of Swadlow and Waxman (1976) for recognizing unmyelinated central axons are applied to axon histograms based on ultrastructural data (Waxman \& Swadlow, 1976) and conduction-velocity measurements (Swadlow \& Waxman, 1976). Electrophysiological analyses seriously underestimate the proportion of very fine-caliber axons known on ultrastructural grounds to be present in cerebral white matter. Moreover, in antidromic stimulation studies, the stimulating electrodes would seldom have initiated antidromic conduction right at the axonal terminal, and therefore the portion of the axonal arborization with the lowest conduction velocity would usually have been omitted from the estimate of axonal conduction delay.

It is thus highly likely that in the cat and the rabbit a significant proportion of cortico-cortical axons have conduction times between soma and synapse approaching $100 \mathrm{msec}$. In larger-brained animals, in which the length of cortico-cortical axons is greater, it is almost certain that even longer conduction delays are imposed by single axons. These intervals are long by usual electrophysiological standards. When measured on the time scale of psychological events, they are brief, but by no means insignificant. The idea that conduction delays in fine-caliber axons may have significance for information processing in the brain has been suggested before (Braitenberg, 
1967). The increase in knowledge in the neurosciences since Braitenberg's suggestion makes it possible to construct more definite hypotheses using this premise.

Given the fact that some cortico-cortical axons do impose such substantial delays on signal transmission, it is certain that the population of axons connecting any two cortical areas will have a wide variety of conduction velocities, consequently imposing a wide range of delays between soma and synapse. These delays would include the more familiar ones of only a few milliseconds as well as the very long delays mentioned in the previous paragraphs. It is therefore probable that a hypothetical signal reaching the cortex from subcortical structures at an instant in time will spread its influence upon other cortical projection areas over a briefly extended interval of time, rather than an instant of time. Conversely, any neuron in the projection area may receive a simultaneous convergence of inputs that had originated at the periphery dispersed over a similar brief interval of time. If such neurons were capable of selecting the synaptic connections to which they are responsive (as presumbly happens in examples of pattern learning for instantaneous patterns), single neurons would be able to encode the conjunction of temporally dispersed signals that defines a temporal pattern.

\section{The Application of the Hebbian Paradigm to Encoding of Brief Temporal Patterns}

In the above paragraphs, I have argued that the variety of conduction delays for the connections in the cerebral white matter are anatomically capable of encoding brief temporal patterns in the single neurons on which they converge. There remains the physiological question of how signals become associated as they converge simultaneously on single neurons. The immediate answer to this is provided by the Hebbian principle for synaptic modification, according to which synapses are strengthened if there is simultaneous presynaptic and postsynaptic activity in the neuron (Hebb, 1949). Since the postsynaptic neuron will be more likely to fire if several presynaptic inputs are activated together than if just one is active, it follows that synapses will tend to be strengthened in groups, the same groups that tend to converge upon and simultaneously activate such neurons. This principle has been thoroughly investigated in regard to pattern learning for simultaneous patterns (e.g., visual patterns; see, e.g., Bienenstock, Fregnac, \& Thorpe, 1983; Cynader \& Chernenko, 1976; Hirsch \& Spinelli, 1970; Schlaer, 1971). The mechanism underlying the Hebbian modification of synapses is under investigation in many laboratories, especially in the form of long-term potentiation (LTP) of synaptic influences (Bliss \& Lomo, 1973), particularly in in vitro slice preparations from the hippocampus (Abraham \& Goddard, 1983; Alger \& Teyler, 1976; Andersen, Sundberg, Sveen, Swann, \& Wigstrom, 1980; Dunwiddie \& Lynch, 1978).
There is one important respect in which the Hebbian principle must be extended if it is to be a basis for encoding brief temporal patterns: The postsynaptic conjunctive process, which allows the strengthening of links of correlated inputs to a neuron, and upon which the Hebbian principle is supposed to depend, must be averaged over a very short interval of time. If this postsynaptic state decayed slowly, say over half a second, its location in time would not be sufficiently precise to pinpoint the instant of convergence of the different components of the temporal pattern. If the proposed mechanism is to operate, the decay time for the postsynaptic influence must be $50 \mathrm{msec}$ or less, since recognition of phonemes and other brief temporally dispersed patterns requires at least this degree of temporal resolution.

In a recent investigation of the Hebbian mechanism in hippocampal slices, Kelso, Ganong, and Brown (1986) studied the postsynaptic conjunctive process directly, by imposing brief intracellular depolarizations at different times in relation to impulse trains in the input pathways. It is clear from Kelso et al.'s results that the conjunctive process represents a temporal window less than $100 \mathrm{msec}$ in duration, but it is not clear exactly how much shorter than this it is. A less direct method, however, gives a more definite indication of the degree of synchrony of converging inputs required for synaptic strengthening. LTP in the hippocampus is usually elicited by activation of quite short pathways (e.g., those from the entorhinal cortex), which may be expected to show little temporal dispersion of signals on their way to the hippocampal neurons in which synaptic modification is detected. Thus, the time course of decay of the conjunctive process can be inferred from the degree of synchrony between two inputs that permits the occurrence of LTP. This method was employed by Levy and Steward (1983) and Gustafsson and Wigstrom (1986). These studies are in agreement that repeated pairing of impulses and/or impulse trains in two different inputs to the hippocampus does not result in LTP unless the interval between impulses in the two afferent lines is less than about $20 \mathrm{msec}$. The temporal characteristics of the Hebbian process are thus sufficiently precise to allow encoding of brief temporal patterns with at least as much accuracy as is observed in behavioral pattern recognition.

\section{Evidence That the Cerebral Cortex Can Encode Repeatedly Presented Temporal Patterns}

Above I presented a hypothesis that the learning of temporal patterns can take place by selection of synapses whose axons impose specified conduction delays upon a signal. Is there any empirical evidence that the suggested process actually occurs? A number of studies in which the neuronal substrate of classical conditioning has been investigated provide such evidence. These results indicate that in the process of elaborating a classically conditioned response, not only does the fact of the association between the conditioned stimulus (CS) and the uncondi- 
tioned stimulus (US) become encoded, but so does the temporal relationship between the two stimuli. Thus, there appears to be a component of the classical conditioning mechanism that can select not only the links of the CS that are coactivated with those of the US, but also those CS links that activate neurons at the particular latency that coincides with the US-induced activation of a neuron. This was demonstrated in three early studies using gross electrographic records of cortical neural activity. Morrell and Jasper (1956) and Yoshii and Hockaday (1958) used a light flickering at a regular frequency of $6-12 \mathrm{~Hz}$ as the US, while a nonrhythmic tone, light, or somatic shock served as the CS. The developing electrographic response to the CS tended to include activity at the same frequency as the US. Chow and Dewson (1964) used neuronally isolated slabs of cortex, with a high-frequency tetanus at one end of the slab as the US and low-frequency $(4-12 \mathrm{~Hz})$ electrical pulses at the other end as the CS. They showed that the developing conditioned response (CR) tended to encode the rhythm of the CS. This could be shown by shifting the CS frequency, when the CR tended to retain the rhythmicity of the old CS. (For example, following a shift from $8 \mathrm{~Hz}$ to $4 \mathrm{~Hz}$, activity was seen not only in time with each pulse of the stimulus train, but also bisecting the interpulse interval.) Voronin, Gerstein, Kudryashov, and Ioffe (1975) used direct cortical stimuli to the motor cortex as the US and clicks or flashes as the CS, which developed the capacity to elicit the same motor response as was elicited by the US. This CR was monitored electromyographically. It was sometimes observed that when the US was omitted, a CR was nevertheless produced, and it was locked to the expected time of the US. More recently, several single-unit studies have demonstrated similar phenomena. Rosenblum and $O$ 'Brien (1977) showed that changes in the response of single cortical units during conditioning applied only to certain parts of the poststimulus time histogram (PSTH). If the thalamic ventralis anterior nucleus was cooled, certain parts of this PSTH reverted to baseline, and, concomitantly, the conditioning of these specific parts of the PSTH was abolished. Both Voronin (1980) and Shevko and Bakanova (1981) reported on a number of neurons whose conditioned responses were also time-locked to the US position. Rusinova (1980), who used cortical stimuli as the CS and US, gave details of the latency of the CR for three different values of the CS-US interval. As this interval was lengthened from 0 to 100 to $150 \mathrm{msec}$, the CR latency also lengthened, from 40 to $100-200$ to $200 \mathrm{msec}$. Thus, although the CR did not exactly coincide with the expected arrival of the US, there was a strong trend for it to approximately coincide. O'Brien and Fox (1969) produced a more complex set of results. Excitatory-unit CRs were time-locked to the US (as in the above studies), whereas inhibitory-unit CRs were time-locked to the CS. In summary, it is clear that the classical conditioning process can encode temporal relationships between the CS and the US, or the temporal properties of either of these stimuli.

\section{Encoding of Brief Temporal Patterns and Phoneme Recognition by the Human Forebrain}

The arguments presented in the previous section can be interestingly applied to the neural basis of language. It is a striking biological fact about language processing in the human brain that the dominant hemisphere (usually the left) is specialized for many language functions. This lateral specialization undoubtedly has many facets; one that has been identified is the sensory ability to recognize consonant speech sounds (phonemes). If the corticocortical connections of the human cerebrum have caliber spectra similar to those in animals, there is an anatomical repertoire of "delay lines" by which such temporal patterns can be encoded. The particular pathways that are likely to encode such temporal patterns are those linking primary auditory areas (where frequency-specific neurons are found) with association areas in which connections from several such frequency-specific neurons may converge upon single neurons. Since phoneme recognition is an acquired rather than an innate ability, a learning mechanism such as that outlined in the previous paragraphs seems also to be required.

The capacity to recognize consonant phonemes is one of the facets of language processing that are performed better by the left hemisphere than by the right. This superior ability appears to reflect the left hemisphere's better capacity for analysis of brief temporal patterns in the auditory mode (Studdert-Kennedy \& Shankweiler, 1970; Tallal \& Schwartz, 1980). It is thus an attractive possibility that the left hemisphere's superiority for phoneme discrimination depends on differences in conductionvelocity characteristics of the population of cortico-cortical axons between the left and right hemispheres.

The specific hypothesis is advanced here that the relevant population of fine-caliber axons has a wider range of axonal calibers, and hence of conduction velocities, on the left side than on the right, thereby permitting wider temporal divergence/convergence of signals from frequency-specific neurons in the left than in the right auditory cortex. Exactly which population of axons this hypothesis applies to is uncertain. It could apply to the whole of the white matter originating in the left side, or, alternatively, to more restricted populations originating from the left cortical areas concerned with sensory analysis of speech (i.e., the left primary auditory area and/or Wernicke's area).

\section{Indirect Evidence Compatible With the Postulated Difference Between Left and Right Hemispheres in Man}

Electrophysiological studies of averaged auditory evoked potentials (AEPs) or of the EEG have generally not been designed to detect the sort of interhemispheric difference postulated above. In at least one study of auditory AEPs, however, a left/right difference has been seen that is compatible with the postulate. Molfese (1978) 
recorded the AEPs produced by consonant sounds ("ba" and " $g a$ ") and resolved them into six factors by principalcomponents analysis, followed by Varimax rotation. One of the factors so derived (with a peak at 70 msec latency) discriminated significantly between the two speech sounds when records from the left hemisphere were used, but not when those from the right were used. Thus, such an analysis using data from the left hemisphere permitted determination of which speech sound had been delivered. This was not possible with data from the right hemisphere.

A more specific pointer in favor of the present hypothesis comes from work on the EEG by Thatcher, Krause, and Hrybyk (1986). These workers computed interareal coherence (correlation between two EEG traces recorded from different sites at the same time) and phase lag (delay, in milliseconds, of one trace with respect to the other at which computed values of coherence were greatest). These two values were computed at various frequencies, for EEGs recorded from different parts of the left and right hemispheres of humans. Thatcher et al. found that the right hemisphere tended to show stronger interareal coherence, whereas interareal phase lag was greater in the left hemisphere. They related the first of these findings to recent evidence that the right hemisphere in man has a higher proportion of white to gray matter than does the left (Gur et al., 1980). Thatcher et al. suggested that the right hemisphere has more long connections than the left, resulting in greater interareal coherence of the EEG. There is, however, an alternative explanation that more parsimoniously accounts for the findings of both Thatcher et al. and Gur et al. This is that the left hemisphere contains less white matter not because it has numerically fewer long axons, but because its axons tend to be finer in caliber than those in the right hemisphere. This would result in the greater phase lag observed in the left hemisphere, and, if it is assumed that interareal conduction is faster and temporally less dispersed in the right than in the left hemisphere, could account for the better interareal coherence seen in the EEGs from the right hemisphere.

\section{Approaches to Direct Testing of the Hypothesis}

The most direct test of the hypothesis concerning the biological basis of the left-hemisphere advantage for phoneme recognition involves actual microscopic analysis of cerebral white matter in man. Ultrastructural techniques would certainly be needed, since the finest-caliber axons are those of critical importance. Ideally, samples of white matter from the left and right hemispheres of the human would be subjected to electron microscopic analysis, so that axonal caliber spectra could be obtained. This suggestion is, however, beset by considerable technical and ethical difficulties. Among the technical difficulties is the need to discriminate reliably between profiles of unmyelinated axons and those of glial cell processes. Probably the most plausible way to accomplish the experiment would involve use of tissues obtained during surgical temporal lobectomy, the important comparison being made between two groups of tissue samples, obtained, respectively, from left and right lobes, from a series of cases. This approach is nevertheless far from ideal: the pathological processes that make such an operation necessary may introduce unwanted factors into the comparison between tissues from left and right. In addition, there would be considerable difficulty in obtaining representative samples.

As an alternative to this approach, there is the possibility of using tissues from experimental primates, with which it would be feasible to use all the necessary technical procedures to ensure good quality electron micrographs and to overcome the sampling problems. In at least one species of monkey (Macaca fuscata) there appears to be a left-hemisphere dominance (similar to that found in humans) for recognition of the species' naturally produced "speech" sounds (Petersen, Beecher, Zoloth, Moody, \& Stebbins, 1978).

\section{Summary and Comment}

The general theme of this paper is that neural signals in the cortex are dispersed temporally as they spread through cortico-cortical pathways, because of the variation in conduction delays between axons. Such variation in the delay lines linking different cortical areas is a suitable substrate for the representation of patterns that are extended briefly in time (over intervals up to about $100 \mathrm{mec}$, or more in the larger-brained primates). The Hebbian principle is sufficiently temporally precise to select synapses whose convergent activations in a neuron are synchronous to within $20 \mathrm{msec}$. Therefore the network of cortico-cortical connections, and their putative modifiable synapses, is capable of acquiring representations of brief temporal patterns present in the sensory input. This hypothesis was discussed with reference to sensory analysis such as may be found early in the processing of an auditory signal; it may be applied specifically to the acquisition of cerebral representation of consonant speech sounds in the human left hemisphere. Suggestions were made as to the biological basis of this hemispheric specialization. This hypothesis derives initially from an application of the principles discussed here to monosynaptic links in the forebrain. However, it appears probable that much of the brain's representation of meaning consists of cell assemblies (Hebb, 1949) in which multisynaptic connections and feedback loops are commonplace, rather than single cells with only monosynaptic, feedforward connections. In such configurations there are no doubt reasons other than those discussed above to explain why signals impinging on the cortical network should be temporally dispersed-for instance, relay along multisynaptic rather than monosynaptic pathways, or reverberatory activity in recursive loops. The application of the principles discussed in the present paper to such configurations of neural connections introduces fresh complications. For instance, how can a multisynaptic pathway retain specificity of representation when there is the possibility of divergence/convergence of signals at the intermediate synap- 
tic nodes? Furthermore, how can pattern representation in cell assemblies be used to re-create temporal sequences in memory recall, as well as to recognize them as an input function? These are large questions, which apply to the representation of simultaneous patterns as well as temporal patterns. Moreover, it is by no means clear what might be their relevance to interhemispheric differences. It is possible, however, that in such cell assemblies patterns that are extended temporally to a greater extent than the 100-200 msec interval discussed here may be similarly encoded.

\section{REFERENCES}

Abraham, W. C., \& Goddard, G. V. (1983). Asymmetric relationship between homosynaptic long-term potentjation and heterosynaptic long-term depression. Nature, 305, 717-719.

AlgER, B. E. \& TEYLER, T. J. (1976). Long-term and short-term plasticity in the CA1, CA3 and dentate regions of the rat hippocampal slice. Brain Research, 110, 463-480.

Andersen, P., Sundberg, S. H., Sveen, O., Swann, J. W., \& WigSTROM, H. (1980). Possible mechanisms for the long-term potentiation of synaptic transmission in hippocampal slices from guinea pig. Journal of Physiology, London, 302, 463-482.

Bienenstock, E., Fregnac, Y., \& ThORPE, S. (1983). Iontophoretic clamp of activity in visual cortex neurones in the cat: A test of Hebb's hypothesis. Joumal of Physiology, London, 345, 123P

Buss, T. V. P., \& Lomo, T. (1973). Long-lasting potentiation of synaptic transmission in the dentate area of the anaesthetized rabbit following stimulation of the perforant path. Joumal of Physiology, London, 232, 331-356.

Braitenberg, V. (1967). Is the cerebellar cortex a biological clock in the millisecond range? In C. A. Fox \& R. S. Snider (Eds.), Progress in brain research: Vol. 25. The cerebellum (pp. 334-346). Amsterdam: Elsevier.

Chow, K. L., \& Dewson, J. H. (1964). Bioelectrical activity of isolated cortex: I. Responses induced by interaction of low- and highfrequency electrical stimulation. Neuropsychologia, 2, 153-165.

Cynader, M., \& Chernenko, G. (1976). Abolition of direction selectivity in the visual cortex of the cat. Science, 193, 504-505.

DuNwidpre, T., LYNCH, G. (1978). Long-term potentiation and depression of synaptic responses in the rat hippocampus: Localization and frequency dependency. Joumal of Physiology. London, 276, 353-367

Ferraya Moyano, H., \& Molina, J. C. (1980). Axonal projections and conduction properties of olfactory peduncle neurons in the rat. Experimental Brain Research, 39, 241-248.

FleischHauer, K., \& WartenberG, H. (1967). Elektronenmikroscopische Untersuchungen über das Wachstum der Nervenfasern und über das Auftreten von Markschneiden im Corpus Callosum der Katze. Zeitschrift für Zellforschung, 83, 508-581.

Gur, R. C., Packer, I. K., Hungerbuhler, J. P., Reivich, M., Obrist, W. D., Amarnek, W. S., \& SackheIm, H. A. (1980). Difference in distribution of gray and white matter in cerebral hemispheres. Science, 207, 1226-1228.

GUSTAFsSON, B., \& WigStrom, H. (1986). Hippocampal long-lasting potentiation produced by pairing single volleys and brief conditioning tetani in separate afferents. Journal of Neuroscience, 6, 1575-1582.

HaUg, H., Kölls, M. \& Rast, A. (1976). The postnatal development of myelinated nerve fibres in the visual cortex of the cat. Cell \& Tissue Research, 167, 265-288.

HEBв, D. O. (1949). The organization of behavior: A neurophsychological theory. New York: Wiley.

Hirsch, H. V. B., \& SPINelLi, D. N. (1970). Visual experience modifies distribution of horizontally and vertically oriented receptive fields in cats. Science, 168, 869-871.

Kelso, S. R., Ganong, A. H., \& Brown, T. H. (1986). Hebbian syn- apses in hippocamopus. Proceedings of the National Academy of Science, 83, 5326-5330.

Lee, K. H., Chung, K., Chung, J. M., \& Coggeshall, R. E. (1986) Correlation of cell body size, axon size and signal conduction velocity for individually labelled dorsal root ganglion cells in the cat. Joumal of Comparative Neurology, 243, 335-346.

LEVY, W. B., \& STEWARD, O. (1983). Temporal contiguity requirements for long-term associative potentiation/depression in the hippocampus. Neuroscience, 8, 791-797.

MilleR, R. (1976). Distribution and properties of commissural and other neurons in the cat sensorimotor cortex. Journal of Comparative Neurology, 164, 361-373.

MILlER, R. (1981). Meaning and purpose in the intact brain. Oxford, England: Oxford University Press.

MoLfESE, D. L. (1978). Left and right hemisphere involvement in speech perception: Electrophysiological correlates. Perception \& Psychophysics, 23, 237-243.

MORRELL, F., \& JASPER, H. H. (1956). Electrographic studies of the formation of temporary connections in the brain. Electroencephalography \& Clinical Neurophysiology, 8, 201-215.

Naito, H. Miyakawa, F., \& Ito, N. (1971). Diameters of callosal fibres interconnecting cat sensorimotor cortex. Brain Research, 27, 369-372.

O'Brien, J. H., \& Fox, S. (1969). Single cell activity in the cat motor cortex: I. Modifications during classical conditioning procedures. Journal of Neurophysiology, 32, 267-286.

Petersen, M. R., Beecher, M. D., Zoloth, S. R., Moody, D. B., \& Stebirins, W. C. (1978). Neural lateralization of species-specific vocalization by Japanese macaques (Macaca fuscata). Science, 202 , 324-327.

RemaHL, S., \& Hrcoebrand, C. (1982). Changing relation between onset of myelination and axon diameter range in developing feline white matter. Joumal of Neurological Science, 54, 33-45.

Rosenblum, S. M., \& O'Brien, J. H. (1977). A cryogenic study of cortical conditioning changes. Journal of Neurophysiology, 40, 957-967.

Rusinova, E. V. (1980). Role of intervals between conditioned and unconditioned stimuli in formation of a cellular analog of temporary connection. Neuroscience \& Behavioral Physiology, 10, 387-393.

SCHLAER, R. (1971). Shift in binocular disparity causes compensatory changes in the cortical structure of kittens. Science, 192, 801-803.

SEGGIE, J., \& BERRY, M. (1972). Ontogeny of interhemispheric evoked potentials in the rat: Significance of myelination of the corpus callosum. Experimental Neurology, 35, 215-232.

Shevko, G. N., Bakanova, N. F. (1981). Dynamics of single unit activity in the association cortex of waking cats during defensive conditioning. Neuroscience \& Behavioral Physiology, 11, 286-292.

Studdert-KenNedy, M., \& Shankweiler, D. (1970). Hemispheric specialization for speech perception. Journal of the Acoustical Society of America, 48, 579-594.

SwadLow, H. A. (1974). Properties of antidromically activated callosal neurons and neurons responsive to callosal input in rabbit binocular cortex. Experimental Neurology, 43, 424-444.

SwaDLOW, H. A., \& WAXMAN, S. G. (1976). Variations in conduction velocity and excitability following single and multiple impulses of visual callosal axons in the rabbit. Experimental Neurology, 53, 128-150.

Swadlow, H. A., Waxman, S. G., \& Geschwind, N. (1980). Small diameter non-myelinated axons in primate corpus callosum. Archives of Neurology, 37, 114-115.

SwadLow, H. A., \& WeYAND, T. G. (1981). Efferent systems of the rabbit visual cortex: Laminar distribution of the cells of origin, ax onal conduction velocities and identification of axonal branches. Journal of Comparative Neurology, 203, 799-822.

TAllal, P., \& SChwarTz, J. (1980). Temporal processing, speech perception and hemispheric asymmetry. Trends in Neuroscience, 3 , 309-311.

TAYLOR, C. A. (1969). Physics and music. Proceedings of the Royal Institute of Great Britain, 42, 237-252.

Thatcher, R. W., Krause, P. J., \& Hrybyk, M. (1986). Cortico- 
cortical associations and EEG coherence: A two-compartment model. Electroencephalography \& Clinical Neurophysiology, 64, 123-143.

VoroniN, L. L. (1980). Microelectrode analysis of the cellular mechanisms of conditioned reflex in rabbits. Acta Neurobiologiae Experimentalis, 40, 335-370.

Voronin, L. L., Gerstein, G. L., Kudryashov, I. E., \& IofFe, S. V. (1975). Elaboration of a conditioned reflex in a single experiment with simultaneous recording of neural activity. Brain Research, 92, 385-403.

WAXMAN, S. G., \& SWADLOW, H. A. (1976). Ultrastructure of visual callosal axons in the rabbit. Experimental Neurology, 53, 115-127.

Wiggins, R. C., Bissell, A. C., Durham, L., \& Samorajski, T. (1983). The corpus callosum during post-natal undernourishment and recovery: A morphometric analysis of myelin and axon relationships. Brain Research, 328, 51-57.

Willey, J. T., Maeda, G., \& Rafuse, D. (1975). Antidromic units in the prepyriform cortex driven by olfactory peduncular volleys. Brain Research, 92, 132-136.

YoshII, N., \& HockadAY, W. J. (1958). Conditioning of frequencycharacteristic repetitive electroencephalographic response with intermittent photic stimulation. Electroencephalography \& Clinical Neurophysiology, 10, 487-502.

(Manuscript received March 6, 1987; revision accepted for publication July 29,1987$)$. 